\title{
Putative therapeutic targets in the treatment of visceral hyperalgesia
}

\author{
S Collins
}

Gut 2004;53(Suppl II):ii19-ii21. doi: 10.1136/gut.2003.033456

The management of abdominal pain remains a major challenge for clinicians despite an explosion of knowledge regarding the physiology and pathophysiology of sensory neural circuits. Initial progress was made in the field of somatic pain and this provided broad hypotheses that could be tested in the field of visceral pain. The major advances in our understanding of basic mechanisms underlying visceral pain originated in the urinary tract and have been applied to the gut. As yet, this increased understanding of enteric sensory physiology has yet to generate new effective treatments for abdominal pain. This review addresses novel insights into peripheral mechanisms underlying visceral hyperalgesia and their applicability to the development of novel therapeutic approaches to the treatment of chronic abdominal pain.

Correspondence to: S Collins, Division of Gastroenterology, Room 4W8, McMaster University Medical Centre, Hamilton, Ontario L8N 3Z5, Canada; scollins@mcmaster.ca

Accepted for publication 16 October 2003
$\mathrm{T}$ he commonest clinical setting of abdominal pain is in the area of functional bowel disorders, and irritable bowel syndrome (IBS) in particular. This syndrome is heterogeneous not only in its clinical presentation, but also in its underlying pathophysiology and pathogenesis. Pain is a dominant feature of IBS and has been largely attributed to visceral hyperalgesia. ${ }^{1}$ However, not all patients with IBS exhibit demonstrable hyperalgesia on formal testing. This may reflect an alternative mechanism for pain generation or shortcomings in experimental design and application. Traditionally, research in IBS has focused on behavioural issues and there is a belief that pain reporting in IBS reflects altered central processing of sensory information, increased vigilance, or illness behaviour. ${ }^{2}{ }^{3}$ However, IBS patients do not exhibit a generalised increase in sensitivity to pain and studies indicate that somatic pain thresholds in IBS patients are either normal or increased. ${ }^{45}$

There is increased acceptance that events in the gastrointestinal tract, such as acute infection, trigger IBS $^{6}$ and there is a suspicion that low grade inflammation ${ }^{7}$ or immune activation ${ }^{8}$ may serve to maintain gut dysfunction which, in turn, provides a basis for symptom generation-much like asthma. This workshop on Visceral Hypersensitivity focused on advances in our understanding of sensory physiology, with emphasis on the mechanisms underlying the transduction of noxious stimuli and the sensitisation of sensory nerves. Whereas in some instances data were provided from extra gastrointestinal systems such as the urinary tract, or from in vitro systems such as dorsal root ganglia cultures, the principles derived from these studies provide a conceptual framework for our understanding of the pathophysiology of chronic abdominal pain and for the design of novel therapeutic approaches.

Basic scientific studies of sensory nerves emphasise the dynamic and flexible state of sensory input and these features are usually implied in the term "neural plasticity". It is within the plasticity of sensory nerves that the challenges and therapeutic opportunities lie. Receptors that transduce stretch or changes in tension in the wall of the gut are well described and their importance lies mainly in the subconscious regulation of motor function. The notion that IBS is a primary motility abnormality has lost is former appeal but should not be entirely discarded. While abnormal motor patterns are non-specific, they certainly occur in some IBS patients and likely reflect altered enteric neurotransmission rather than a primary abnormality of smooth muscle or the interstitial cells of Cajal. If this is indeed the case, it is possible that the altered neurotransmitter environment could affect nociception. This is plausible in light of new findings regarding the intraganglionic laminar ending (IGLE), which is a basket-like structure that surrounds myenteric ganglia and which represents a nociceptor of vagal afferents in the gut. ${ }^{9}$ If the enteric nervous system is a primary site of abnormality in IBS, it is conceivable that the altered chemical milieu of myenteric ganglia could sensitise the IGLE and contribute to visceral hyperalgesia. In this regard, it is important to note that a recent report, utilising laparoscopically assisted full thickness biopsies from patients with "severe IBS", identified lymphocytic infiltration of myenteric ganglia. ${ }^{10}$

The processes underlying the sensitisation of sensory nerves have been the subject of a large number of studies, many of which have been summarised in the workshop. In broad terms, the chemical sensitisation of sensory nerves has been classified on the basis of either selective or modality specific sensitisation or promiscuous sensitisation. The former represents the sensitisation of sensory nerves by distinct sources and

\footnotetext{
Abbreviations: IBS, irritable bowel syndrome; IGLE, intraganglionic laminar ending; $E E$, enteroendocrine; $E C$, enterochromaffin; 5-HT, 5-hydroxytryptamine; PAR-2, protease activated receptor-2; VGSC, voltage gated sodium channel; TTXr, tetrodotoxin resistant; NGF, nerve growth factor; IBD, inflammatory bowel disease
} 
mediators such as the release of cholecystokinin from enteroendocrine (EE) cells or 5-hydroxytryptamine (5-HT) from enterochromaffin cells (EC) in the gut. ${ }^{11}$ The putative role of 5-HT and EC cells in IBS has received much attention and the development of 5-HT3 receptor antagonists have had limited success in the treatment of abdominal pain in IBS. ${ }^{12}$ Promiscuous sensitisation refers to the release of mediators following inflammatory or ischaemic injury. Examples include the release of arachidonic acid metabolites and cytokines from inflammatory cells as well as the release of tryptase from mast cells. The latter is of potential importance to the pathophysiology of IBS as increased numbers of mast cells have been identified in some IBS patients. The activation of the protease activated receptor-2 (PAR-2) on primary afferents by mast cell derived tryptase could be a mechanism of sensitisation in IBS. PAR-2 receptors are G protein coupled receptors, the ligands for which are trypsin and mast cell tryptase. They have been identified on several cell types in the gut including primary afferents. ${ }^{13}$ Increased numbers of mucosal mast cells have been reported in IBS. ${ }^{14}{ }^{15}$ Thus, mast cell activation in IBS could be a mechanism for inducing hyperalgesia and PAR-2 antagonists could offer therapeutic benefit.

Investigation of the molecular bases for sensitisation of afferent nerves is well underway and has revealed a wide array of mechanisms and mediators. The bases for sensitisation induced by neurotransmitters and trophic agents, temperature, and protons are currently being identified. There is particular interest in the cation selective ion channel vanilloid receptor (VR-1) and its subtypes, ${ }^{16}{ }^{17}$ and it is evident that activation of these receptors is not restricted to heat and protons, but may occur with a variety of chemicals including lipid ligands. There is also much interest in the family of voltage gated sodium channels (VGSC) and in the VGSC $\alpha$ subunit SNS Navl.8 which is a tetrodotoxin resistant (TTXr) channel thought to be present in dorsal root ganglia and small diameter neurons and which is activated during inflammatory events in the gut. ${ }^{18}$ Nerve growth factor (NGF) sensitises sensory nerves through peripheral mechanisms as well as through altered gene expression. Of considerable clinical interest are the observations that NGF is elevated in conditions such as interstitial cystitis and IBD, and that NGF can induce persistent as well as transient sensitisation. ${ }^{19}$ The role of NGF in IBS remains to be determined.

There is a rebirth of interest in the role of bacteria in IBS. The putative involvement of microbes ranges from pathogenic bacteria causing acute gastroenteritis leading to postinfective IBS, and the putative role of flora in this condition. Manipulation of flora with probiotics may alter sensory perception, as suggested by preliminary reports of improvement in abdominal pain and bloating in IBS patients. ${ }^{20}{ }^{21}$ In this context, it is important that we understand how bacteria might influence the sensory apparatus. The most superficial sensory nerve endings lie in the mucosa but do not impinge on the gut lumen. Signals from the lumen must, therefore, be transduced by cells in the epithelial lining. The roles of EC and EE cells in sensitisation have been discussed. Bacteria are known to interact with intestinal epithelial cells to generate mediators that include cytokines, which in turn can diffuse and sensitise sensory nerve endings. Bacteria also influence intestinal permeability, facilitating access of chemicals from the lumen to the gut wall. Direct interaction between bacterial products and primary afferents has been demonstrated in the context of cholera toxin..$^{22}$

The role of non-granular "brush cells" in sensitisation remains speculative, but similarities between these cells and those responsible for taste, via the $G$ coupled protein gustducin, raise the possibility that these cells may act as chemosensors of luminal content and may sensitise sensory nerves via the release of nitric oxide. ${ }^{23}$ The "M cell" at the dome of Peyer's patches in the gut is responsible for antigen uptake and presentation to immune cells in the "traffic area" of Peyer's patches. Recent work using various nervous system specific markers including protein gene product 9.5, neuron specific enolase, neurofilament 200, S-100 protein, and the glial fibrillary acidic protein, has shown there is an intimate and specific structural association between enteric nerves and the compartments of Peyer's patches, including the dome. Nerves are located around the follicle in the traffic and dome areas, providing an infrastructure whereby information regarding lumenal antigen can be transduced to the nervous system via the immune system. ${ }^{24}$ Studies on viral uptake indicate that this circuitry involves vagal pathways to the brain. ${ }^{25}$ This new interface warrants further investigation as another aspect of the modulation of sensory information in the periphery.

In summary, new and exciting data are emerging regarding the sensitisation of sensory nerves. Understanding of these mechanisms will provide unique opportunities for the development of antinociceptive strategies. These include drugs to inhibit the actions of sensitising agents as well as via the manipulation of intestinal flora. Further work is required to exploit endogenous mechanisms that reduce the excitability of sensory nerves, as this may offer broader antinociceptive therapy. Cannabinoid receptors offer a potential therapeutic target in this regard..$^{26} \mathrm{~A}$ list of putative targets for the treatment of hyperalgesia, based on this presentation, is shown in table 1.

There are also some concerns. The majority of studies addressing visceral hypersensitivity use models of acute and often severe inflammation. In contrast, the human conditions that require new approaches are chronic and more subtle in their expression of inflammation. Thus, advances based on these paradigms need to be examined in chronic models of low grade inflammation or selective immune activation. In addition, closer attention needs to be paid to the nature of the inflammatory stimulus and response used in these experiments. As we learn more about the sensory modulatory characteristics of inflammatory and immune mediators, it is likely that different inflammatory infiltrates will confer different influences on the sensory apparatus. By refining the inflammatory stimuli, it may be possible to determine whether chronic inflammation induces persistent hyperalgesia and whether certain types of chronic inflammation produce hypoalgesia, as has been suggested based on observation in patients with chronic IBD. ${ }^{27}$

Table 1 Putative therapeutic targets for visceral hyperalgesia

\begin{tabular}{ll}
\hline Target & Ref \\
\hline 5-HT3 receptor & 11 \\
CCK receptor & 12 \\
NK antagonists & 19 \\
Voltage gated sodium channels on sensory nerves & 18 \\
(Nav1.8 subunit) & 19 \\
Cyclo-oxygenase and other inflammatory mediators & 13 \\
PAR-2 receptors & 16,17 \\
VR-1 receptor & 26 \\
Cannabinoid receptor & 19 \\
NGF & 20,21 \\
Intestinal bacteria (via probiotics/antibiotics); no published & \\
data, see refs & \\
\hline
\end{tabular}




\section{REFERENCES}

1 Mayer EA, Raybould HE. Role of visceral afferent mechanisms in functional bowel disorders. Gastroenterology 1990;99:1688-704.

2 Naliboff BD, Munakata J, Chang L, et al. Toward a biobehavioral model of visceral hypersensitivity in irritable bowel syndrome. J Psychosom Res 1998;45:485-92.

3 Silverman DH, Munakata JA, Ennes $\mathrm{H}$, et al. Regional cerebral activity in normal and pathological perception of visceral pain. Gastroenterology 1997;1 12:64-72

4 Accarino AM, Azpiroz F, Malagelada JR. Selective dysfunction of mechanosensitive intestinal afferents in irritable bowel syndrome [see comments]. Gastroenterology 1995; 108:636-43.

5 Cook IJ, van Eeden A, Collins SM. Patients with irritable bowel syndrome have greater pain tolerance than normal subjects. Gastroenterology 1987:93:727-33.

6 Rodriguez LA, Ruigomez A. Increased risk of irritable bowel syndrome after bacterial gastroenteritis: cohort study. BMJ 1999;318:565-6.

7 Collins SM, Piche T, Rampal P. The putative role of inflammation in the irritable bowel syndrome. Gut 2001;49:743-5.

8 Chadwick VS, Chen W, Shu D, et al. Activation of the mucosal immune system in irritable bowel syndrome. Gastroenterology 2002;122:1778-83.

9 Grundy D. Neuroanatomy of visceral nociception: vagal and splanchnic afferent. Gut 2002;51(Suppl I):12-15.

10 Tornblom H, Lindberg G, Nyberg B, et al. Histopathological findings in the jejunum of patients with severe irritable bowel syndrome. Gastroenterology 2000:118(Suppl I):A140.

11 Hillsley K, Grundy D. Serotonin and cholecystokinin activate different populations of rat mesenteric vagal afferents. Neurosci Lett 1998;255:63-6.

12 Humphrey PP, Bountra C, Clayton N, et al. Review article: the therapeutic potential of 5-HT3 receptor antagonists in the treatment of irritable bowel syndrome. Aliment Pharmacol Ther 1999:13(Suppl 2):31-88.

13 Vergnolle N, Wallace $\mathrm{JL}$, Bunnett NW, et al. Protease-activated receptors in inflammation, neuronal signaling and pain. Trends Pharmacol Sci $2001 ; 22: 146-52$.
14 Weston AP, Biddle WL, Bhatia PS et al. Terminal ileal mucosal mast cells in irritable bowel syndrome. Dig Dis Sci 1993;38:1590-5.

15 O'Sullivan M, O Morain CA. Increased mast cells in the irritable bowel syndrome. Neurogastroenterol Motil 2000 Oct;12:449-57.

16 Benham CD, Davis JB, Randall AD. Vanilloid and TRP channels: a family of lipid-gated cation channels. Neuropharmacology 2002;42:873-88.

17 Gunthorpe MJ, Benham CD, Randall A, et al. The diversity in the vanilloid (TRPV) receptor family of ion channels. Trends Pharmacol Sci 2002; 23: 183-91.

18 Kerr BJ, Souslova V, McMahon SB, et al. A role for the TTX-resistant sodium channel Nav 1.8 in NGF-induced hyperalgesia, but not neuropathic pain. Neuroreport 2001;12:3077-80

19 McMahon SB. NGF as a mediator of inflammatory pain. Philos Trans $R$ Soc Lond B Biol Sci 1996;351:431-40.

20 Nobaek S, Johansson ML, Molin G, et al. Alteration of intestinal microflora is associated with reduction in abdominal bloating and pain in patients with irritable bowel syndrome. Am J Gastroenterol 2000;95:1231-8.

21 Pimentel M, Chow EJ, Lin HC. Eradication of small intestinal bacteria overgrowth reduces symptoms of irritable bowel syndrome. Am J Gastroenterol 2000;95:3503-6.

22 Kirchgessner AL, Tamir H, Gershon MD. Identification and stimulation by serotonin of intrinsic sensory neurons of the submucosal plexus of the guinea pig gut: activity-induced expression of Fos immunoreactivity. J Neurosci 1992; 12:235-48.

23 Hofer D, Asan E, Drenckhahn D. Chemosensory perception in the gut. News Physiol Sci 1999; 14:18-23.

24 Krammer HJ, Kuhnel W. Topography of the enteric nervous system in Peyer's patches of the porcine small intestine. Cell Tissue Res 1993;272:267-72.

25 Morrison LA, Sidman RL, Fields BN. Direct spread of reovirus from the intestinal lumen to the central nervous system through vagal autonomic nerve fibers. Proc Natl Acad Sci U S A 1991;88:3852-6.

26 Iversen L, Chapman V. Cannabinoids: a real prospect for pain relief? Curr Opin Pharmacol 2002;2:50-5.

27 Bernstein CN, Niazi N, Robert M, et al. Rectal afferent function in patients with inflammatory and functional intestinal disorders. Pain 1996;66:151-61. 